\title{
Observations of high-velocity SAPS-like flows with the King Salmon SuperDARN radar
}

\author{
A. V. Koustov ${ }^{1}$, R. A. Drayton ${ }^{1}$, R. A. Makarevich $^{2}$, K. A. McWilliams ${ }^{1}$, J.-P. St-Maurice ${ }^{1}$, T. Kikuchi ${ }^{3}$, and \\ H. U. Frey ${ }^{4}$ \\ ${ }^{1}$ Institute of Space and Atmospheric Studies, University of Saskatchewan, 116 Science Place, Saskatoon, S7N 5E2, Canada \\ ${ }^{2}$ Department of Physics, La Trobe University, Victoria 3086, Australia \\ ${ }^{3}$ Solar-Terrestrial Environment Laboratory, University of Nagoya, 3-13 Honohara, Toyokawa, Aichi 442, Japan \\ ${ }^{4}$ Space Sciences Laboratory, University of California, Berkley, CA 94720-7450, USA
}

Received: 14 September 2005 - Revised: 20 April 2006 - Accepted: 12 May 2005 - Published: 3 July 2006

\begin{abstract}
In this study, a focused investigation of the potential for the King Salmon (KS) SuperDARN HF radar to monitor high-velocity flows near the equatorial edge of the auroral oval is undertaken. Events are presented with lineof-sight velocities as high as $2 \mathrm{~km} / \mathrm{s}$, observed roughly along the L-shell. Statistically, the enhanced flows are shown to be typical for the dusk sector (16:00-23:00 MLT), and the average velocity in this sector is larger (smaller) for winter (summer) conditions. It is also demonstrated that the highvelocity flows can be very dynamical with more localized enhancements existing for just several minutes. These shortlived enhancements occur when the luminosity at the equatorial edge of the auroral oval suddenly decreases during the substorm recovery phase. The short-lived velocity enhancements can be established because of proton and ion injections into the inner magnetosphere and low conductance of the ionosphere and not because of enhanced tail reconnection. This implies that some KS velocity enhancements have the same origin as subauroral polarization streams (SAPS).
\end{abstract}

Keywords. Ionosphere (Plasma convection: Ionospheric irregularities: Auroral ionosphere)

\section{Introduction}

Observations of ionospheric plasma convection at subauroral latitudes showed the occasional occurrence of extremely fast flows of more than $2-3 \mathrm{~km} / \mathrm{s}$ (e.g. Galperin et al., 1973; Anderson et al., 1991, 1993, 2001; Yeh et al., 1991; Karlsson et al., 1998; Foster and Vo, 2002; Carpenter and Lemaire, 2004). Such flows are believed to be stretched in the magnetic $\mathrm{L}$ shell direction over a significant portion of the dusk ionosphere (Anderson et al., 1991; Foster and Vo, 2002). Some of the flows are very strong and localized in

Correspondence to: A. V. Koustov

(sasha.koustov@usask.ca) latitude, covering less than half a degree of magnetic latitude (Anderson, 1991) while others are less intense but more extended, spanning over several degrees of magnetic latitude (Yeh et al., 1991; Foster and Vo, 2002). Events were identified for which the flow exhibited two maxima in its latitudinal profile; one enhancement at relatively high latitudes, around the expected location of the auroral oval, and the other one at much lower latitudes (Spiro et al., 1979; Yeh et al., 1991; Anderson et al., 2001). It was suggested to use a common term, sub-auroral polarization streams (SAPS), to characterize enhanced flows equatorward of the auroral oval (Foster and Burke, 2002). It is believed that SAPS occur because of energetic ion and proton injections into the inner magnetosphere/plasmasphere and the setting up of radial polarization electric fields. An important factor in establishing a strong polarization electric field is the low conductance of the ionosphere at subauroral latitudes, so that the Region 2 fieldaligned currents driven by particle pressure in the inner magnetosphere can be closed through perpendicular ionospheric currents and thus support a strong northward electric field and westward convection of the ionospheric plasma. These processes are more frequent during magnetic storms (e.g. Galperin, 2002; Carpenter and Lemaire, 2004). Persistent correlation of SAPS with substorm occurrence has also been reported (e.g. Anderson et al., 1993; Galperin, 2002), but it is generally expected that any tail convection enhancement that accelerates particles can potentially lead to SAPS formation (e.g. Anderson, 2001; Ridley and Liemohn, 2002).

SAPS have been traditionally studied with the Defense Meteorological Satellite Program (DMSP) satellite ion drift and precipitation data. However, satellite measurements are rather episodic, as it takes approximately $100 \mathrm{~min}$ for a satellite to return to the same latitude, and the combination of three satellites often does not help because of a large longitudinal separation between them. In this respect, SAPS monitoring with ground-based radars is advantageous because these measurements can be taken continuously.

Published by Copernicus GmbH on behalf of the European Geosciences Union. 


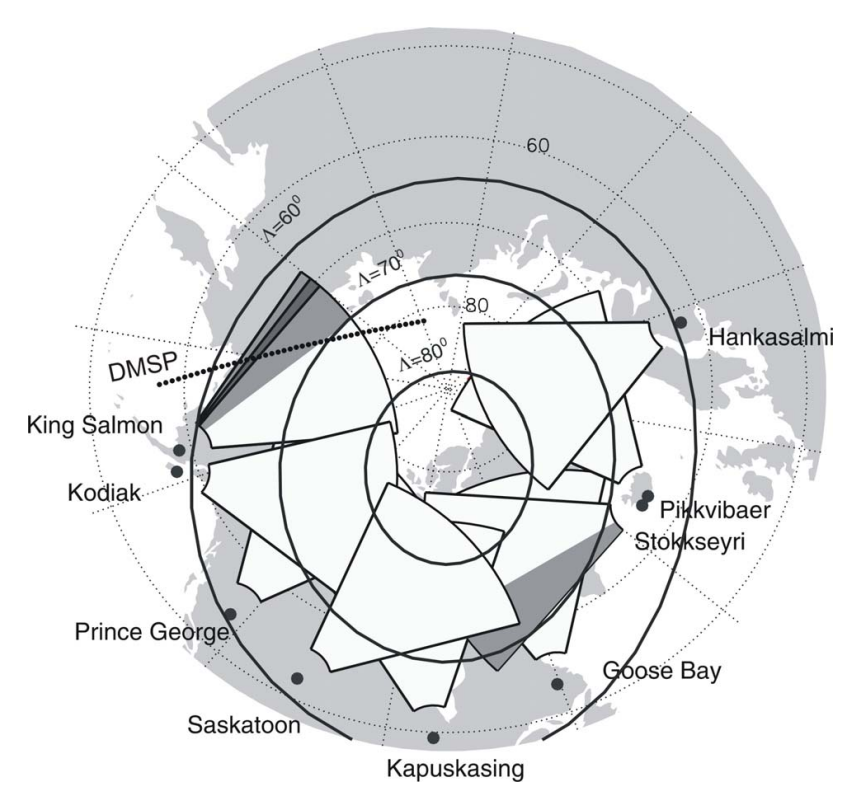

Fig. 1. Fields of view (FoV) of the Northern Hemisphere SuperDARN radars (between 400 and $2800 \mathrm{~km}$ ), the part of the King Salmon (KS) radar observational area (light shading, beams 0-5) from where the measurements were statistically analyzed in this study and footprints (at a height of $300 \mathrm{~km}$ ) of DMSP F15 satellite for a pass near the KS FoV. The darker shading within the King Salmon FoV indicates the orientation of beam \#1. Light shading within the Stokkseyri radar FoV shows the area (beams 0-5) from where the measurements were statistically analyzed for a comparison with the KS data.

In the past, the Millstone Hill (MH) incoherent scatter radar has been successfully used to study SAPS (Yeh et al., 1991; Foster and Vo, 2002; Erickson et al., 2002; Foster et al., 2005). Another opportunity to study SAPS is to utilize the Super Dual Auroral Radar Network (SuperDARN) HF radars (Greenwald et al., 1995). Although these radars have been designed to monitor plasma convection at auroral oval and polar cap latitudes, some of them can be used for sub-auroral studies. Parkinson et al. (2003b, 2005a, b) presented examples of high-velocity observations near the equatorward edge of the auroral oval. The authors introduced a term "westward flow channels" to characterize such flows and showed that they are ultimately related to the substorm development in the area of observation. All three studies are based on the Bruny Island (BI) radar data in Australia. The authors also presented a couple of examples of observations with the King Salmon (KS) radar in Alaska. Since then, two new, very useful radars started operation, the Unwin radar in the Southern Hemisphere (New Zealand) and the Wallops Island radar in the Northern Hemisphere (USA). Another new SuperDARN HF radar at mid-latitudes (northern Japan) is under construction. Clearly, the introduction of these new low-latitude radars would significantly improve SuperDARN capabilities in SAPS studies.
In this paper we undertake a more focused investigation of the potential of one of the existing radars, the King Salmon radar, in the detection of high-velocity flows near the equatorward edge of the auroral oval. Our goals are a general assessment of the radar performance, an investigation of the location of the high-velocity streams with respect to the auroral oval, as well as the role of substorm processes in exciting such streams.

\section{Geometry of King Salmon observations and statistics for echo occurrence and velocity}

Figure 1 shows the fields of view (FoVs) of the SuperDARN HF radars in the Northern Hemisphere for ranges of 400 to $2800 \mathrm{~km}$. In accordance with the initial purpose of the SuperDARN experiment, the radars can monitor coherent echoes from a broad range of magnetic latitudes $\Lambda=65^{\circ}-85^{\circ}$, which are typical auroral zone and polar cap latitudes. Potentially, one can detect echoes from the lower magnetic latitudes, especially at Hankasalmi, Kodiak and King Salmon, but one has to keep in mind that echoes at low ranges often come from the E region and their velocities can be well below plasma convection velocities in the F region (e.g. Koustov et al., 2005). If F-region data at low latitudes are available, one can estimate the full convection velocity by using the map potential procedure of Ruohoniemi and Baker (1998). One can also make convection velocity estimates by assuming the flow is roughly L-shell aligned and by simply dividing the measured line-of-sight (1-o-s) velocity by the cosine of the L-shell angle. Such a procedure has been used in a number of Millstone Hill papers (e.g. Foster and Vo, 2002) and to some extent in SuperDARN studies (e.g. Parkinson et al., 2003b).

Figure 1 demonstrates the advantages of the KS radar in the detection of high-velocity subauroral echoes; it has beams that are oriented close to the L-shell direction (so that total convection estimates would have minimum uncertainties) and it can detect F-region echoes from latitudes as low as $60^{\circ}$ in low number (most counterclockwise) beams. In Fig. 1, one can recognize that the Stokkseyri and Pykkvibaer radars also have an appropriate "azimuthal" orientation of the beams, but these radars cover latitudes of $\sim 70^{\circ}$, where detection of SAPS flows is very unlikely, for example, Foster and Vo (2002) reported typical SAPS latitudes of $\Lambda<65^{\circ}$.

In this study we consider routine $\mathrm{KS}$ data gathered in the standard mode of operation with 1-min or 2-min scans through 16 beam positions. The KS radar has been in operation since the end of 2001, but for more than a year it experienced hardware problems, so extended periods were not covered by measurements. Figures $2 \mathrm{a}-\mathrm{c}$ show typical echo occurrence rates in MLT-magnetic latitude $(\Lambda)$ coordinates for three different seasons: winter (December 2001), equinox (March 2002) and summer (July 2003). Only data in beams $0-5$ were considered (see shaded area in Fig. 1) 
a)

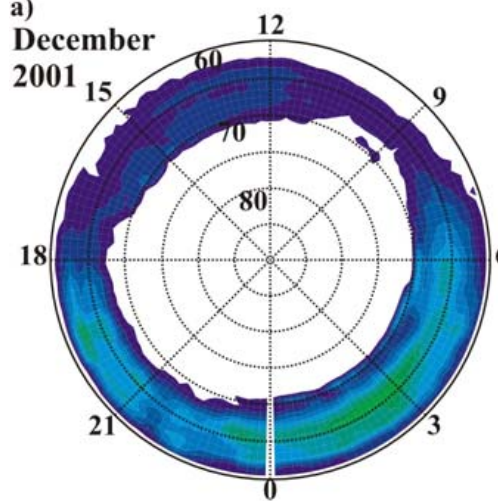

b)

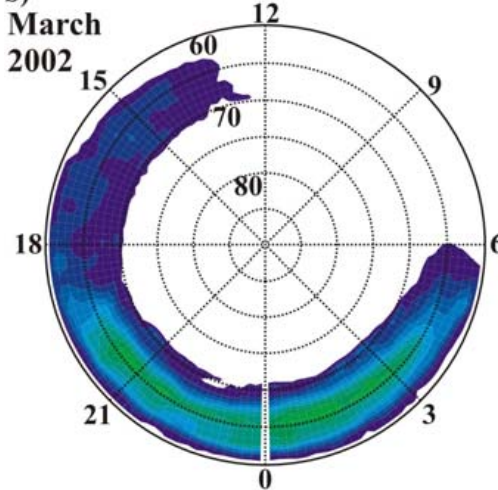

c)

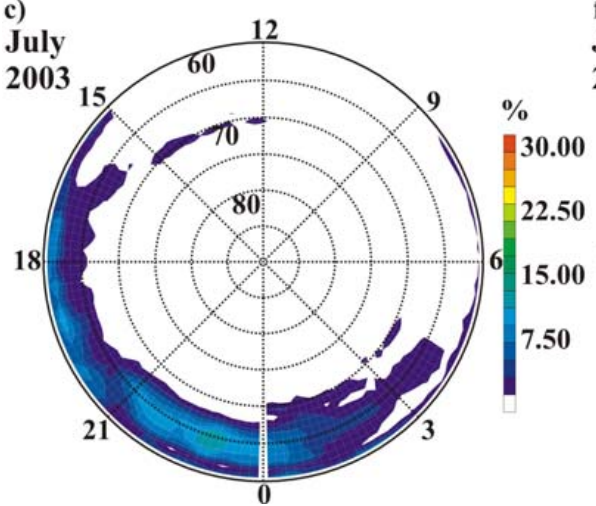

d)

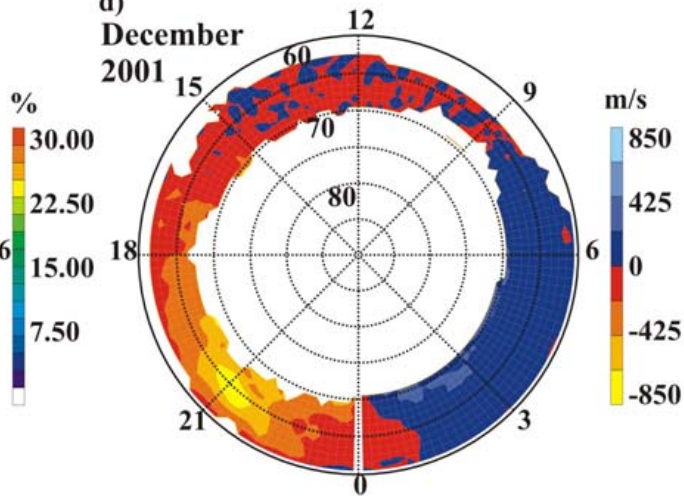

e)

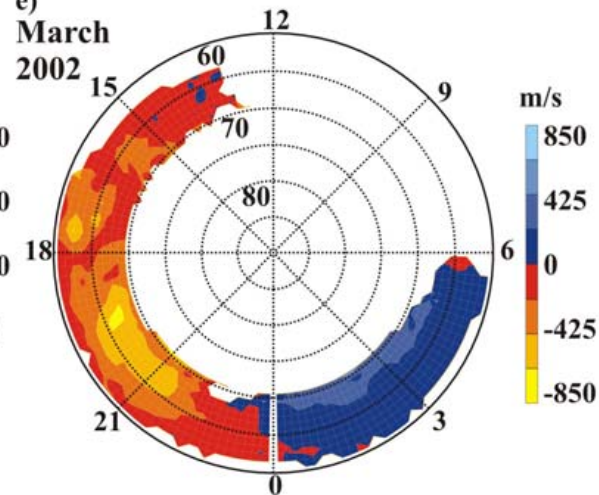

f)

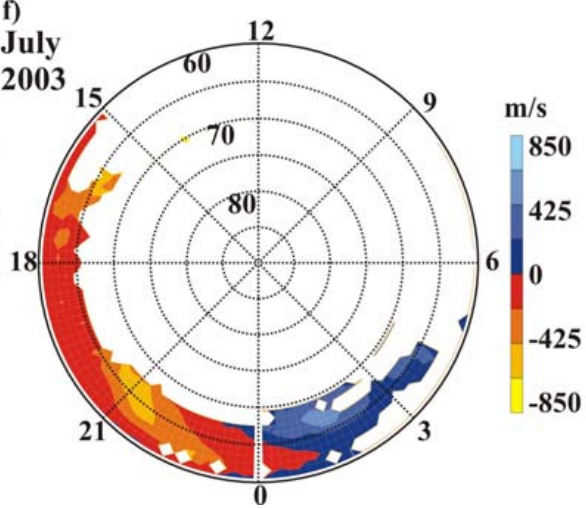

Fig. 2. King Salmon echo occurrence rate and averaged line-of-sight velocity for winter (December 2001, panels (a) and (d)), equinox (March 2002, panels (b) and (e)) and summer (July 2003, panels (c) and (f)) in magnetic latitude - magnetic local time coordinates. Only data in beams $0-5$ were considered.

to concentrate on those directions at which SAPS flows are expected. Occurrence rates were computed as a ratio of the number of registered echoes in every individual radar gate to the total number of observations in this gate for six selected beams over each month. Only ionospheric echoes stronger than $3 \mathrm{~dB}$ having spectral widths $>35 \mathrm{~m} / \mathrm{s}$ and velocities $>30 \mathrm{~m} / \mathrm{s}$ were counted to eliminate untypical echoes and reduce ground scatter contamination. Ratios were averaged over 10 minutes of observations in $1^{\circ}$ magnetic latitude bins. Magnetic local time was computed by taking into account range and universal time. The total numbers of con- sidered scans were 22737 over 17 days in December, 27603 over 24 days in March and 16176 over 24 days in July. The number of scans for each month is larger than the overall number of scans considered by Foster and Vo (2002) with the MH radar. We should mention that we processed all KS data available for 2001-2004 and found that the major features reported below can be identified in more comprehensive statistics. We present data for specific months to avoid possible solar cycle effects (Ruohoniemi and Greenwald, 1997; Koustov et al., 2004). 

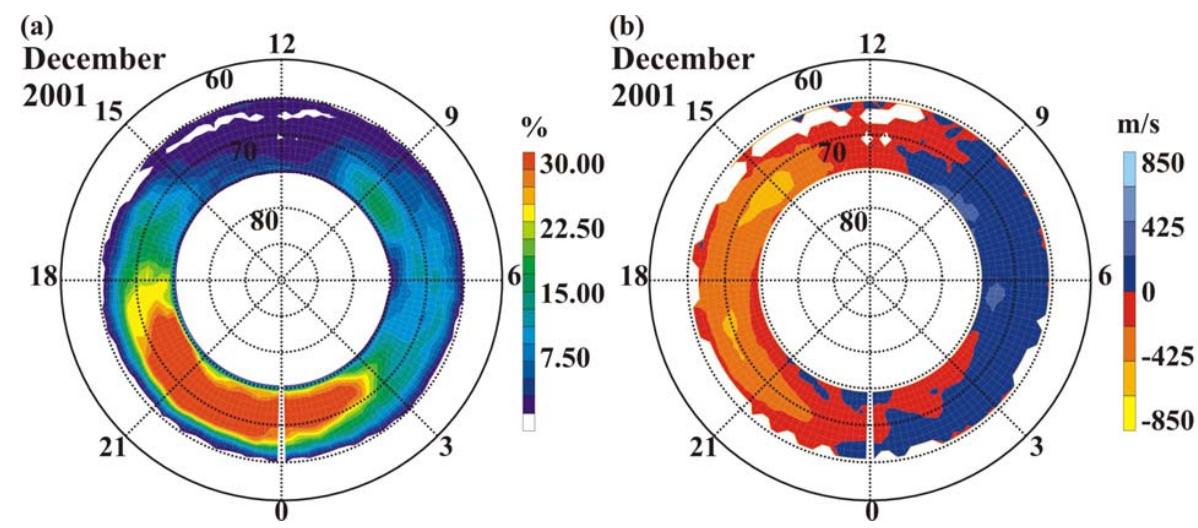

Fig. 3. Stokkseyri echo occurrence rate and averaged line-of-sight velocity for winter (December 2001) in magnetic latitude - magnetic local time coordinates. Only data in beams $0-5$ were considered.

Figures $2 \mathrm{a}-\mathrm{c}$ show that echoes are much more frequent in the nightside sector. Equinox is the preferential season, though winter observations also show significant occurrence rates. Overall echo occurrence rates for the KS radar are lower than for other Northern Hemisphere radars (Koustov et al., 2004). We make appropriate comparison with the Stokkseyri radar data later. The lower occurrence rates might be related to the hardware differences; the KS radar uses a wire antenna that seems to provide less gain.

Figures $2 \mathrm{~d}-\mathrm{f}$ present statistics for the KS 1-o-s velocity in the MLT- $\Lambda$ coordinates. Data are presented only for those bins whose echo occurrence rate was above $1.85 \%$. The same three months of observations are considered. The red (blue) color is used to denote westward, negative (eastward, positive) velocity. The diagrams show a general trend of the velocity polarity reversal around magnetic midnight (red color to blue color) and 09:00 MLT (blue color to red color), as expected. The prominent feature of all three diagrams is a region of strongly enhanced velocities centered around 21:00 MLT and magnetic latitudes of $\sim 65^{\circ}$, seen as a highvelocity "blob" standing out of the background velocity. The absolute values for the maximum average velocity are more than $425 \mathrm{~m} / \mathrm{s}$, with exceptionally strong values of $>640 \mathrm{~m} / \mathrm{s}$ for the winter case, and to a lesser extent, the equinox case. There is a seasonal trend; at certain latitudes, e.g. $\Lambda=65^{\circ}$, large velocities occur in a broader sector of MLT during winter, smaller in equinox and much smaller over summer. In terms of latitude, there is not much variation; the velocity peak seems to be always around $\Lambda=65^{\circ}$. The velocity maximum is achieved at $\sim 21: 00$ MLT for winter and summer and at $\sim 19: 30$ MLT for equinox. We should note that the summer observations show an additional area of very strong velocities between 16:00 and 17:00 MLT at $\Lambda \sim 65^{\circ}$. The enhanced velocities are not accompanied by peaks in the echo occurrence rates, presented in Figs. 2a-c. The processed data for the echo power and spectral width (not presented here) do not show any distinct features associated with the velocity enhancements, indicating that the phenomenon reflects only enhanced flows in the ionospheric plasma.

Data on average KS velocities are in contrast with observations of other SuperDARN radars, which do not show the strong velocity maximum at 21:00 MLT; see, for example, data for all radar beams at Saskatoon and Stokkseyri presented by Hamza et al. (2000). For radars that scan mostly perpendicular to the magnetic $\mathrm{L}$ shells (such as the Saskatoon radar), this lack of a velocity maximum is not a surprise, as the evening sector flow is generally along L-shell directions. This argument does not work for radars whose FoV is "azimuthally" oriented, such as the Stokkseyri radar in Iceland. To illustrate the difference between the KS and Stokkseyri radar velocities, we investigated Stokkseyri observations for the same months as the KS observations (December 2001, March 2002 and July 2003) and computed the occurrence rates and average velocities. The Stokkseyri data for the month of December 2001 (28 941 scans over 31 days) are presented in Fig. 3 in the same format as the KS data in Figs. 2a, d. One can see that the occurrence rate is $2-3$ times larger than for the KS radar and the average velocity is mostly below $425 \mathrm{~m} / \mathrm{s}$ in the evening sector (similar "threshold" value for the KS observations is $\sim 640 \mathrm{~m} / \mathrm{s}$ ). The radar observes at $\sim 5^{\circ}$ larger magnetic latitudes. There is no evidence for the 21:00 MLT velocity peak, with perhaps a possibility that a small peak at $\sim 20$ MLT and $\Lambda=70^{\circ}$ is "a remnant" of the velocity maximum seen by the KS radar at lower latitude and 21 MLT. The average Stokkseyri velocity gradually decreases toward midnight at all magnetic latitudes. Instead of the evening maximum, the Stokkseyri data show a strong velocity peak at $\sim$ 15:00 MLT.

Since average velocities in the evening sector for the KS radar are quite different from velocities for the Stokkseyri radar, which measures at typical auroral oval latitudes, one can hypothesize that the flows observed by the KS radar at lower latitudes can be associated with additional sources that are not operational at auroral zone latitudes. 


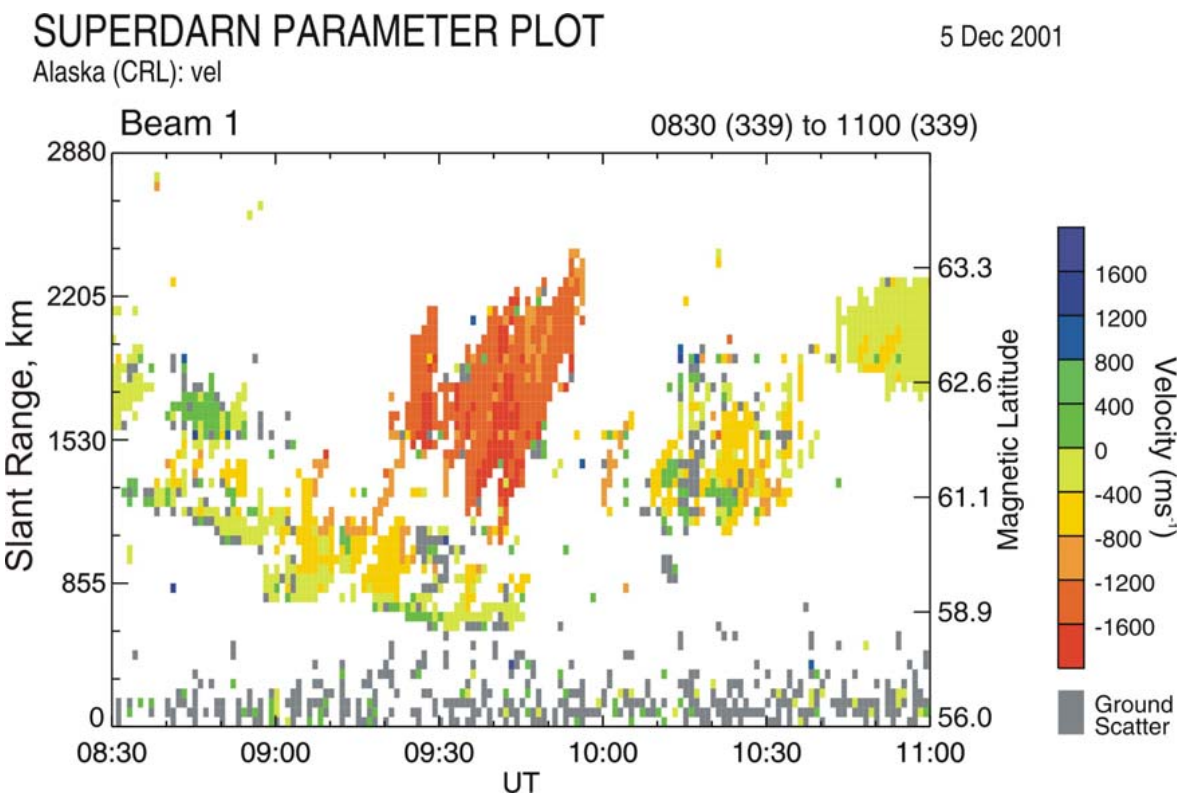

Fig. 4. Echo Doppler velocity in beam 1 of the King Salmon radar for 5 December 2001, 08:30-11:00 UT in range (ACCGM magnetic latitude) - universal time coordinates.

\section{The event of 5 December 2001}

To obtain insights into the reasons for the occurrence of highvelocity KS echoes we analyze one event in detail and identify geophysical conditions corresponding to such an event. We consider data obtained on 5 December 2001.

\subsection{Echo appearance}

Figure 4 presents velocity data in beam 1 for the event of 5 December 2001 between 08:30 and 11:00 UT. For convenience, the 1-o-s velocity is plotted in UT-range and magnetic latitude $\Lambda$ coordinates. One notices the "blob" of echoes with velocity exceeding $1.6 \mathrm{~km} / \mathrm{s}$ at $\Lambda \sim 62.5^{\circ}$ between 09:22 and 09:57 UT. These are exceptionally high velocities for any SuperDARN observations (e.g. Lacroix and Moorcroft, 2000). If one assumes that the flow is L-shell aligned, then the total velocity would be in excess of $2.0 \mathrm{~km} / \mathrm{s}$ (such estimates will be shown later). The event lasted for about $30 \mathrm{~min}$. Echoes with much lower velocity existed prior, during and after the high-velocity blob period, at slightly lower latitudes. These were also F-region echoes (ranges $>900 \mathrm{~km}$ ), thus indicating that the high-velocity echoes were located at latitudes above $\sim 60^{\circ}$. One can notice that the magnitude of the low-velocity echoes increased somewhat at about 09:00 UT and decreased back to low values after 10:00 UT, about 30 min after the high-velocity blob disappeared. In addition, within the highvelocity blob, one can notice a "poleward progression" of the high-velocity echoes. The high-velocity region starts at low latitudes and with time it is seen at progressively higher latitudes. We should note that such a progression is to some ex- tent an apparent effect; analysis of the velocity maps shows that the region of enhanced velocity first appears at smaller ranges and then progresses to larger ranges corresponding to higher magnetic latitudes (the geometry of observations, Fig. 1, is such that the radar beam is "tilted" toward the magnetic pole from the L-shell direction).

We should mention that large velocities were observed not only in "azimuthal" beams, such as beam 1, but also in more "meridional" beams, such as beams 5-10. The velocity in more meridional beams was not as high as in the azimuthal beams but the radar started their detection from about 08:40 UT with a short-lived strong enhancement around 09:00 UT.

3.2 Geophysical conditions and enhanced flow within global convection pattern

First of all, we note that the event occurred at the recovery phase of a minor storm; the Dst was $-30 \mathrm{nT}$ and the Sym$\mathrm{H}$ index was fairly stable for several hours around the interval of interest. The Kp index was $2+$. Figure 5 summarizes other geophysical conditions on 5 December 2001. Figure 5a shows the maximum velocity detected in beam 1 (at any possible range) at every instant of time. We also indicate by a shaded bar the period for which the highvelocity echo blob was seen in beam 1 (c.f., Fig. 4). One can notice that the strongest magnitude of the flow was observed around 09:40 UT, and the gradual velocity magnitude increase started around 09:00 UT. What is special about 09:00 UT is that this moment is very close to the onset of the substorm at 08:57 UT (as identified from the IMAGE 


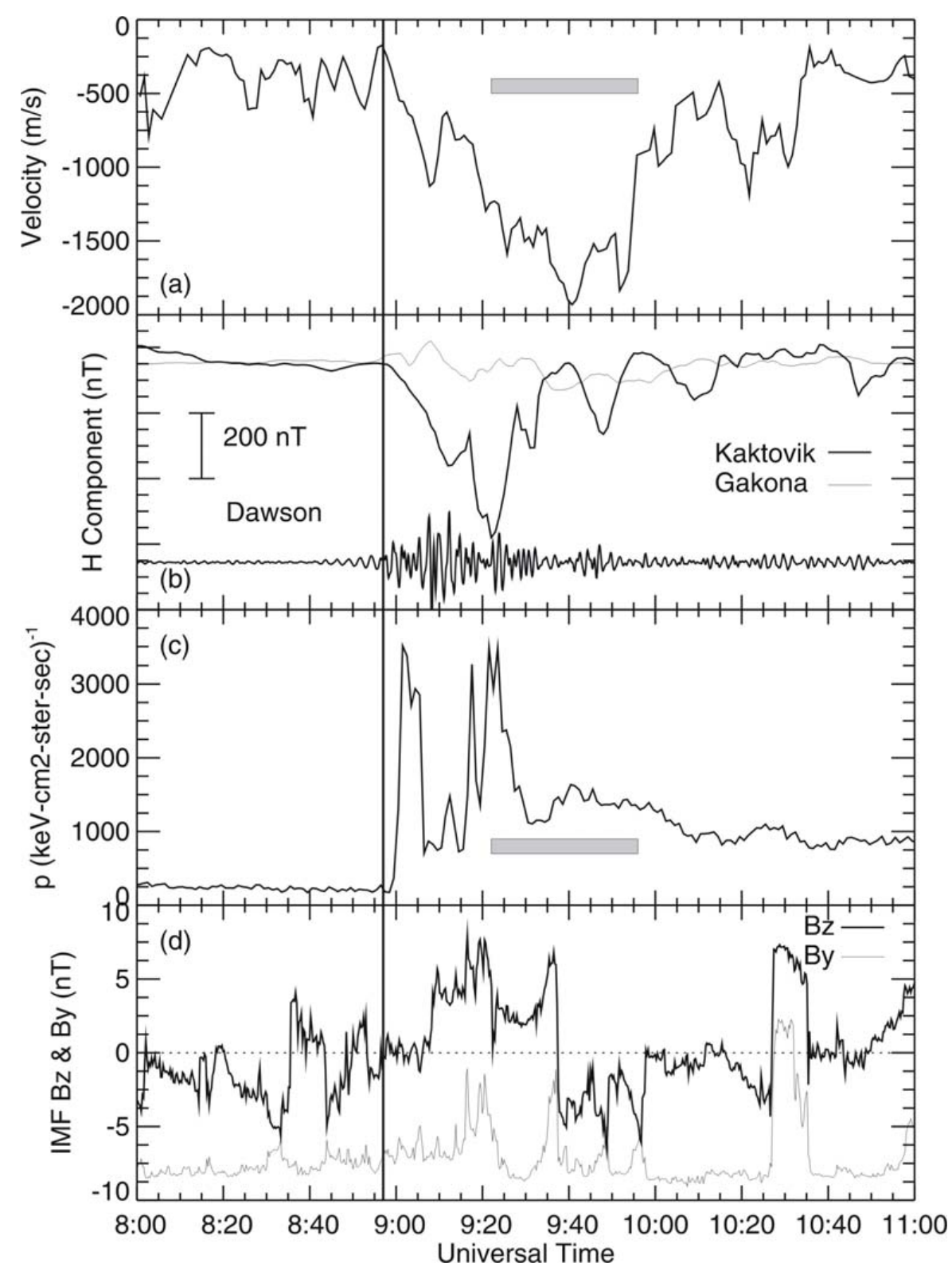

Fig. 5. Summary of the data for the 5 December 2001 event: (a) maximum velocity along the King Salmon HF radar beam 1 (the shaded bar indicates the period for which the high-velocity echo blob was seen on Fig. 4), (b) H-component of magnetic perturbations at Kaktovik and Gakona and Pi2 magnetic pulsations at Dawson (the length of the vertical scale bar corresponds to $33 \mathrm{nT}$ for this trace), (c) flux of energetic protons according to measurements by the geostationary satellite LANL 1991-080 and (d) IMF $B_{z}$ and $B_{y}$ components. Vertical line indicates the substorm onset.

satellite optical data, some of which will be presented later) in the area to the east of the KS radar's FoV. We indicate the substorm onset on all panels of Fig. 5 by the vertical line.

In Figs. 5b, c we present data documenting the substorm development. Figure $5 \mathrm{~b}$ shows the magnetometer observations to the east from the area of high-velocity blob observations, over Alaska (mutual locations of the three magnetometers used will be shown later in Fig. 9). According to the Kaktovik magnetometer, located at a magnetic latitude of $\Lambda=70.7^{\circ}$, the $\mathrm{H}$-component started to rapidly decrease at around 08:57 UT. No decrease was detected at Gakona $\left(\Lambda=63.5^{\circ}\right)$, which indicates the latitudinal locality of the substorm. The record at the bottom of Figure $5 \mathrm{~b}$ is the Pi2 magnetic pulsations as observed at Dawson $\left(\Lambda=66.0^{\circ}\right)$, slightly eastward of Kaktovik and Gakona. One can recognize the Pi2 onset at about 08:57 UT. In Fig. 5c we show the flux of energetic protons (50-400 $\mathrm{keV}$ range) as observed by the Synchronous Orbit Particle Analyzer (SOPA) instrument on 

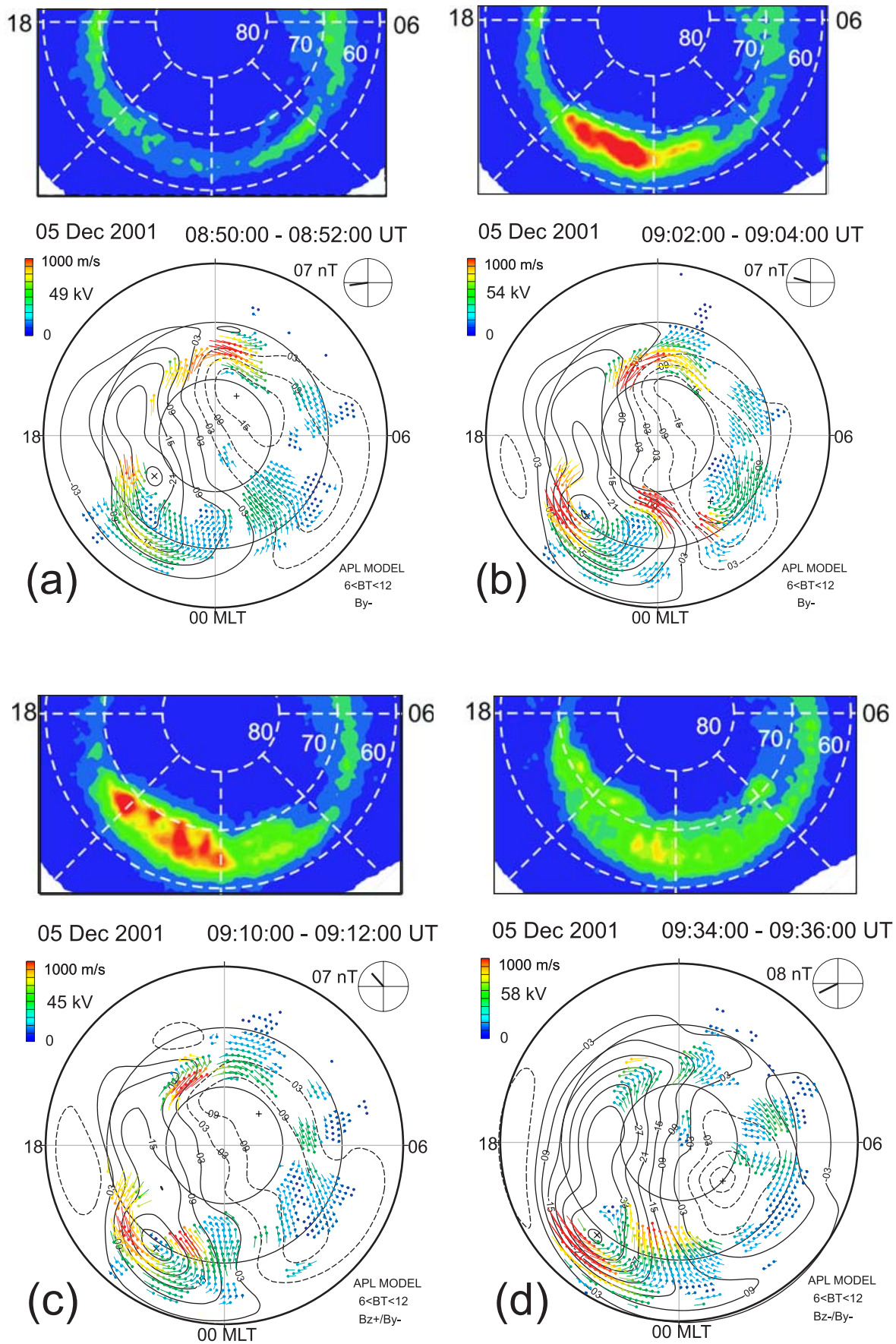

Fig. 6. Four global images of the auroral luminosity according to IMAGE observations and matched SuperDARN maps of plasma convection for a substorm on 5 December 2001. The onset occurred at 08:57 UT, according to IMAGE. Panel (a) corresponds to the growth phase of the substorm ( $\sim 7$ min prior to the onset), panels (b) and (c) correspond to the active phase of the substorm $(\sim 5 \mathrm{~min}$ and $\sim 13 \mathrm{~min}$ after the onset), and panel (d) corresponds to the substorm recovery phase ( $\sim 37 \mathrm{~min}$ after the onset).

the geostationary Los-Alamos National Laboratory (LANL) satellite 1991-080. Over the event duration, the satellite was located in the pre-midnight sector of the magnetosphere (at geographic longitude of $\sim 195^{\circ} \mathrm{E}$, over the area of interest). The flux increases rapidly and strongly around 09:00 UT, in accord with the substorm development. One can recognize two separate intensifications about 20 min apart. These probably correspond to two separate particle injections into the plasmasphere boundary layer. Figure $5 d$ shows the IMF conditions according to the ACE satellite. The $B_{y}$ component 

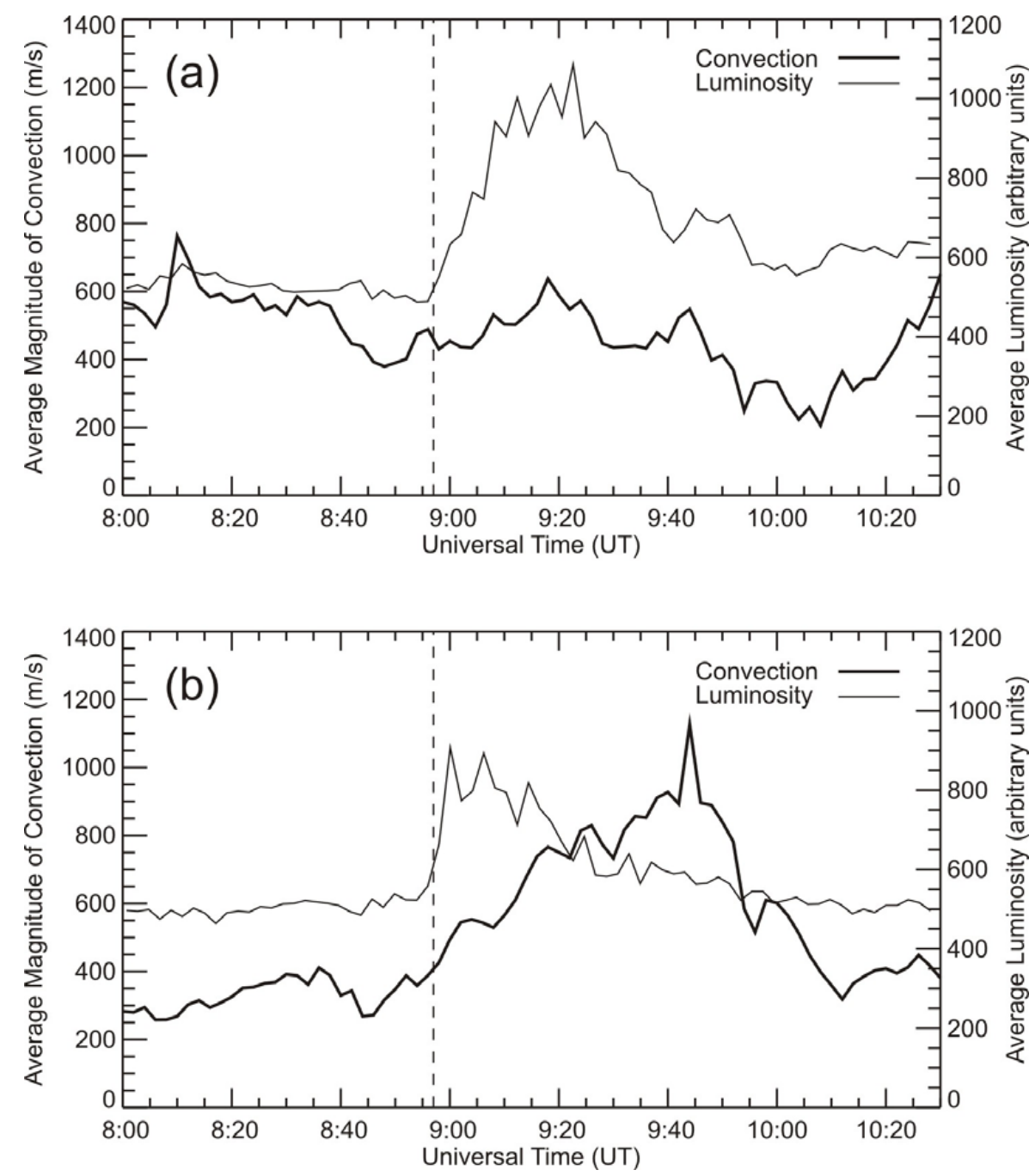

Fig. 7. Average magnitude of the convection and auroral luminosity in the (a) poleward $\left(\mathrm{MLAT}=74^{\circ}-67^{\circ}\right)$ and $(\mathbf{b})$ equatorward $\left(\mathrm{MLAT}=60^{\circ}-\right.$ $67^{\circ}$ ) portions of the auroral oval for the entire evening sector (19:00-23:00 MLT). Vertical dashed line marks the substorm onset.

was mostly negative over the whole event duration. The $B_{z}$ component was around zero for at least $15 \mathrm{~min}$ prior to the onset and it turned northward around the onset time (the IMF data were shifted in Fig. $5 \mathrm{c}$ by $56 \mathrm{~min}$ to account for the disturbance propagation from the satellite position to the magnetosphere).

The final evidence of the substorm development can be inferred from the optical data collected by the IMAGE satellite; the onset is clearly identifiable at 08:57 UT. We do not show IMAGE data around this time; for the purpose of this paper, observations at different times are of more interest.

In Figs. 6a-d we present 4 frames of IMAGE WIC observations at (a) 08:50 UT, (b) 09:02 UT (c) 09:10 UT and (d) 09:34 UT and matched (as close as possible in time) SuperDARN convection maps. The convection maps were obtained by using the standard map potential technique of Ruohoniemi and Baker (1998) and by considering data from all
Northern Hemisphere SuperDARN radars, including the KS radar.

The luminosity and convection maps in Fig. 6a correspond to a quiet oval $\sim 7 \mathrm{~min}$ prior to the substorm onset. The next two panels show the substorm development in the midnight sector. The last panel, Fig. 6d, shows the auroral oval during the substorm recovery phase; these observations correspond to the period of maximum velocity observed at KS.

The SuperDARN maps show some changes in the convection pattern as the substorm progresses. According to Fig. 6a, $\sim 7$ min prior to the substorm onset the convection pattern consisted of two cells with a dominating morning cell, in agreement with the strongly negative $B_{y}$ component of the IMF. The next frame (Fig. 6b) corresponds to the period right after the substorm onset. It clearly shows vortical flows of opposite directions in the dusk and dawn sectors. The vortices are centered at $\Lambda=70^{\circ}$ and 21:00 MLT and 03:00 MLT; 

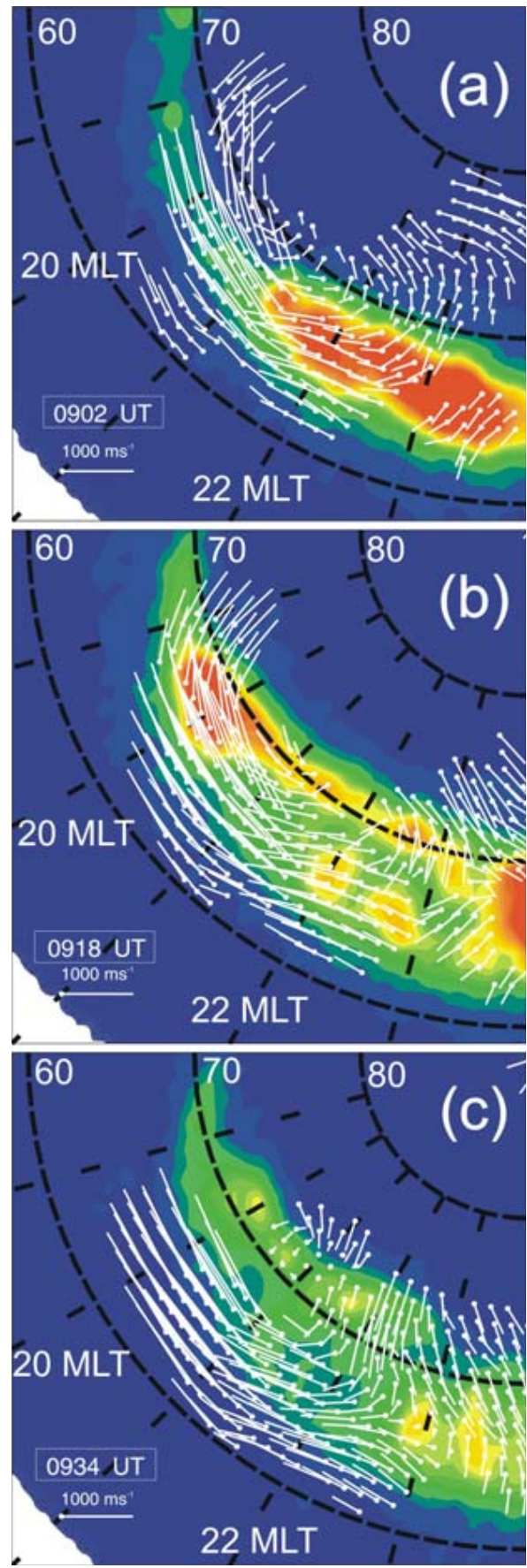

Fig. 8. Auroral luminosity distribution in the dusk sector (IMAGE) and plasma convection (SuperDARN) in magnetic local time - magnetic latitude coordinates. Red (blue) color corresponds to strongest (weakest) luminosity. (a) 09:02 UT. Fast flow is seen in the area westward of the brightest luminosity (auroral bulge) in the magnetic local time sector of 20:00-21:00 MLT. (b) 09:18 UT. Fast flow starts developing in the area equatorward of the brightest luminosity in the magnetic local time sector of 20:00-22:00 MLT. (c) 09:34 UT. Very fast flow is seen in the area equatorward of the brightest luminosity in the magnetic local time sector of 20:00-22:00 MLT. the morning sector vortex is less clear in Fig. 6b. The vortices are very similar to those reported by Grocott et al. (2002) for the substorm onset time. We notice that the flow within the dusk vortex is particularly strong on the west side, in agreement with large KS 1-o-s velocities. The flow at this time has a significant meridional component; it is located at the most westerly edge of the auroral bulge. The enhanced westward flow at the equatorward edge of the evening vortex is also seen as yellow vectors. Figure $6 \mathrm{~d}$ refers to the time about $\sim 15$ min after the substorm onset. Here the dawn vortex seems to have disappeared while the dusk vortex still persists. One can see that the westward flow is now enhanced while the meridional flow weakened. Figure $6 \mathrm{~d}$ shows strong westward flow at the equatorward edge of the dusk vortex that has expanded equatorward with respect to its location in the previous frame. Visual comparison of optical and convection data in Figs. $6 \mathrm{c}$ and $\mathrm{d}$ indicates that the high-velocity westward flow overlaps the equatorward edge of the auroral oval.

Figure 7 gives a quantitative account of changes in the average intensity of the convection (as inferred from 1-min convection maps similar to the ones shown in Fig. 6) and luminosity for the entire evening sector of 19:00-23:00 MLT and for two latitudinal regions, $60^{\circ}-67^{\circ}$ and $67^{\circ}-74^{\circ}$. The first (second) band of latitudes corresponds to the equatorward (poleward) portion of the auroral oval for the event under consideration. The luminosity intensity is presented in relative units. One can see, in Figs. 7a, b, that the luminosity in the equatorward portion of the oval increased abruptly at the substorm onset (vertical dashed line) and decayed significantly in about $20 \mathrm{~min}$. The luminosity in the poleward portion of the oval started to increase near the onset time but reached a maximum $\sim 15 \mathrm{~min}$ later, after the auroral bulge expanded poleward. Convection in the equatorial portion of the oval started to increase $\sim 10$ min prior to the onset and continued to increase until it reached its maximum at around 09:40 UT. Convection in the poleward portion of the oval showed a slight increase about 15 min after the substorm onset. Thus, the average convection in the equatorial portion of the auroral oval shows similar trends as the KS 1-o-s velocity presented in Fig.5a.

\subsection{Enhanced flows and auroral oval location: comparison} with IMAGE and DMSP observations

To explore the mutual location of the enhanced flows and luminosity in detail we plot both data sets on one frame for three moments, 09:02 UT, 09:18 UT and 09:34 UT, Figs. 8a-c. The first and the last moments correspond to observations near the substorm onset time and at the substorm recovery phase when the fastest flows were detected. The additional moment for the comparison was selected because the KS 1-o-s velocity data can be compared with DMSP measurements and also because this frame is one of the first showing a strong westward flow. 


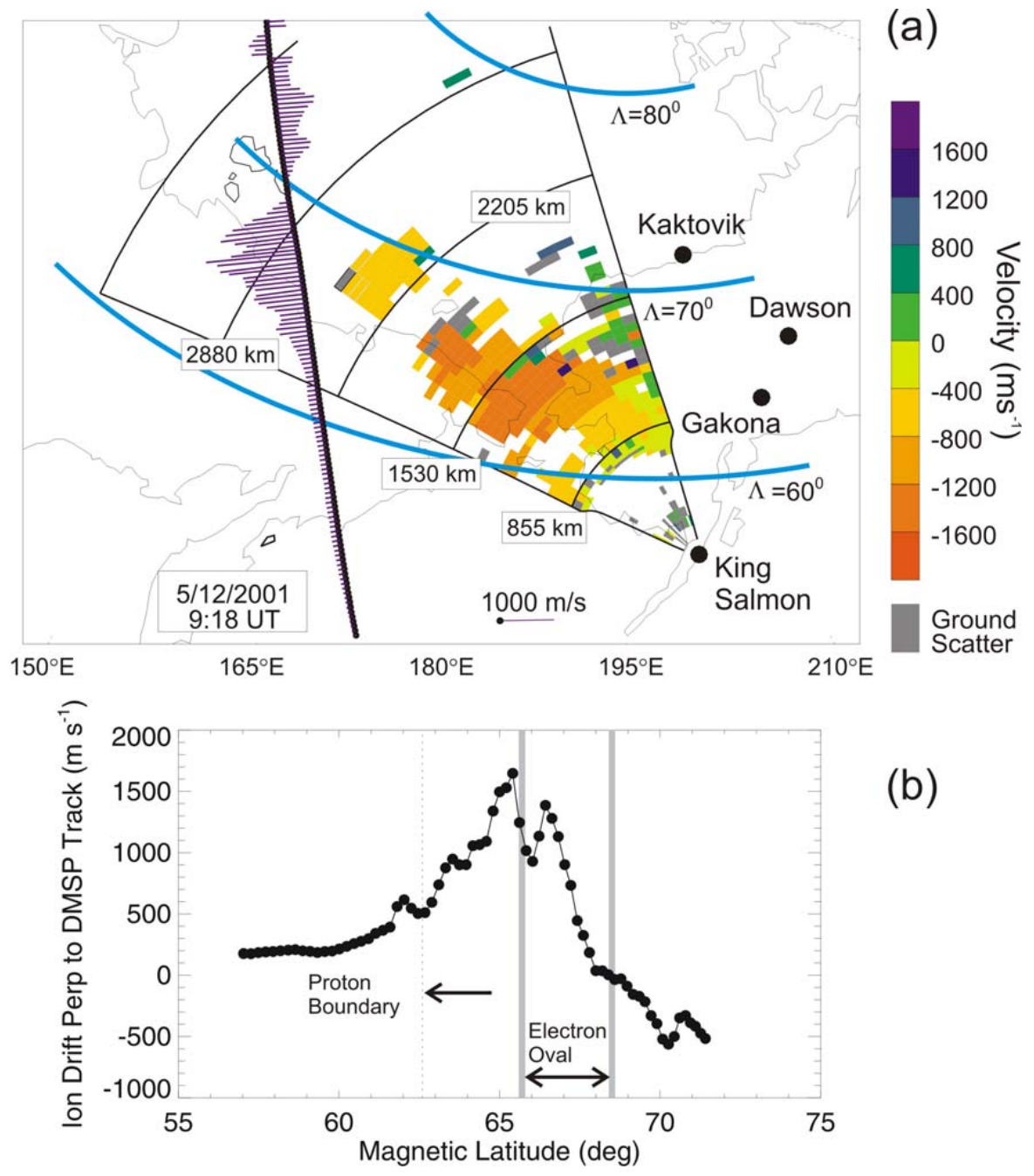

Fig. 9. (a) King Salmon velocity map for 5 December 2001, 09:18 UT with overlaid DMSP cross-track ion drifts for a DMSP F 15 pass near the time of radar measurements. (b) A line plot of the ion drift data shown in panel (a), latitudes of the auroral electron precipitations and the equatorward boundary of the proton aurora. For the boundary identification, DMSP particle data were considered. The peak in the ion drift roughly coincides with the equatorward edge of the electron precipitation and relatively fast flow exists equatorward of this edge for several degrees of magnetic latitude.

First consider the joint IMAGE - SuperDARN observations at 09:02 UT presented in Fig. 8a. One can clearly see the large-scale convection vortex with the focus located to the northwest of the auroral bulge luminosity. The fastest flow is observed to the west of the auroral bulge, and is oriented westward. The flow is also westward (but not as fast) within the bulge area and equatorward (although the amount of vectors located equatorward of the oval is not significant). The enhanced flow correlates with the weaker precipitation/lower conductance to the west of the auroral bulge.

Convection data presented in Fig. 8b for 09:18 UT show the same overall pattern, although the velocity magnitudes are larger. More importantly, the enhanced velocities are now located at lower latitudes in the areas where the lumi- nosity decreased (notice that all diagrams presented in this paper use the same scaling for the luminosity level). Finally, Fig. 8c corresponds to observations at 09:34 UT, the time of the largest westward velocities observed. The flow is clearly located at the very equatorward edge of the luminosity band, well equatorward of the strongest luminosity blobs at MLAT $=70^{\circ}$. This flow can be classified as SAPS, as observationally it satisfies all criteria in terms of the conditions, location and intensity of SAPS.

Because the data presented in Figs. 8a-c have some uncertainties in terms of mapping and spatial resolution of the IMAGE optical instrument, we compare the radar velocity data with observations of the DMSP F15 satellite that passed the KS field of view at about 09:18 UT, as shown in Fig. 9. In 


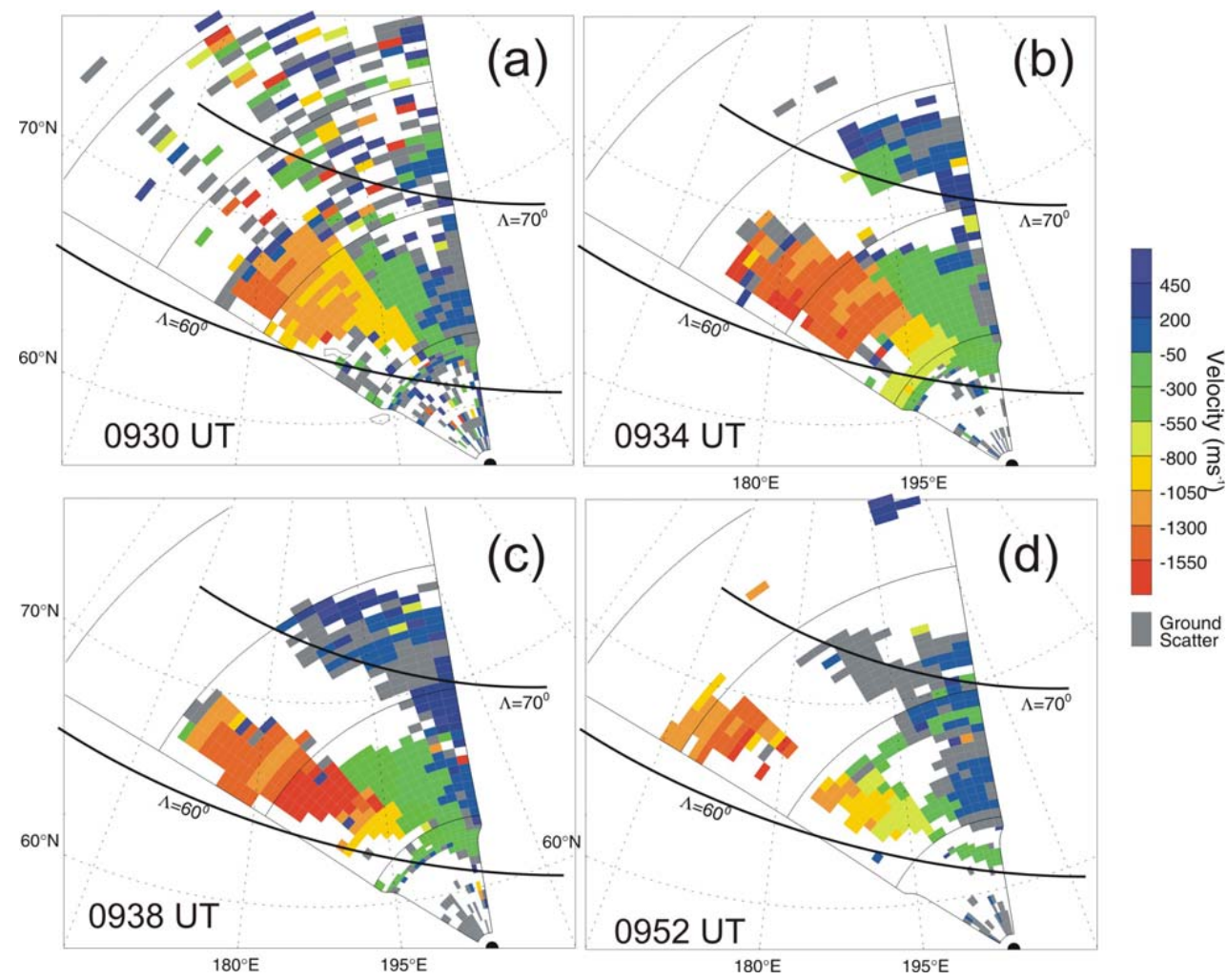

Fig. 10. King Salmon 1-min velocity maps illustrating the onset of very high-velocity echoes roughly along the L-shell directions between $\sim$ 09:34 and 09:38 UT. These echoes correspond to the extremely fast flow identified in Fig. 8c. The transition from the very high velocities to the background ones is seen well in the central beams as a sharp velocity magnitude decrease.

Fig. 9a we present the KS velocity map together with DMSP cross-track velocities. In Fig. 9b we present the DMSP data in more detail and also indicate the location of the equatorward edge of the auroral oval, as determined by the electron precipitation detector on this satellite. The equatorial edge of proton precipitation is also shown. We note that the DMSP velocity maximum is achieved near the equatorward boundary of the electron oval. One can see that the KS high-velocity echoes are located somewhat equatorward of the oval boundary, if one assumes that the oval edge is located at the same latitudes in the areas of the radar and DMSP measurements. As expected, the KS velocities are very comparable with the ion drifts measured by the DMSP (the "look" directions of radar and satellite measurements are about the same). Although the DMSP data show a double maximum in the ion drift velocity while crossing the highlatitude ionosphere, one cannot simply identify similar peaks in the radar data, as there are significant spatial velocity variations, so that more elaborate analysis is required to detect the peaks.

\subsection{On the temporal and spatial variations of the flow speed}

The radar data presented clearly show fast azimuthal flows near the equatorial edge of the auroral oval which can be identified as SAPS-like flow, at least for observations at $\sim 09: 34$ UT. Generally, the observed convection patterns can be affected by several factors, with the most important ones being the external IMF merging-related factors and particle injection-related factors. Separation of the effects of these sources in SuperDARN convection maps is a difficult task. To identify the additional component of the flow pattern outside the auroral oval, the SAPS component, Foster and Vo (2002) considered latitudinal variation of the MH 1-o-s velocity divided by the cosine of the L-shell angle. In presenting the data in this way, they noticed a change in the rate of the velocity variation with latitude (presumably at the "edge" of the oval) or just simply detected a separate stream. Such signatures have been shown to persist during disturbed conditions. Despite the fact that the 5 December 2001 event occurred at relatively quiet conditions $(\mathrm{Kp}=2+)$, we apply a somewhat similar procedure to the KS observations.

We concentrate here on a short interval of observations near 09:34 UT when the fast flows equatorward of the bright blobs of the oval luminosity were detected (as shown in 


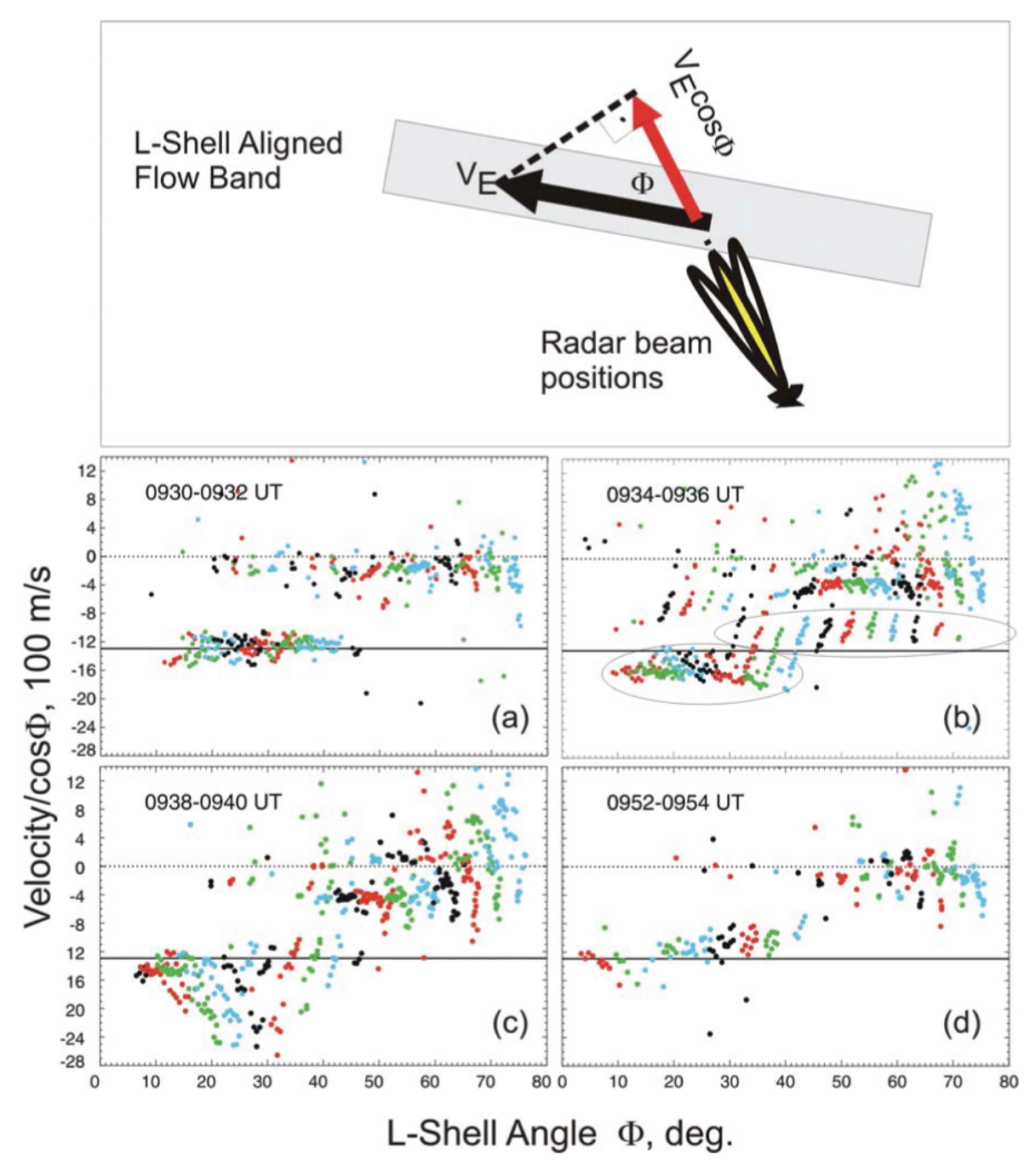

Fig. 11. Top panel: A diagram illustrating the relationship between the measured velocity in SuperDARN observations, the plasma convection velocity $\mathrm{V}_{E}$ and the L-shell angle $\Phi$. Four bottom panels: King Salmon velocity observations in various beams for four 2-min intervals on 5 December 2001 between 09:30 UT and 09:52 UT. The measured line-of-sight velocity was divided by the cosine of the L-shell angle $\Phi$ at every bin of observations. Color code was applied to various beam data as follow: black - beams $0,4,8,12$; red - beams $1,5,9$, 13; green beams 2, 6, 10, 14; and blue - beams 3, 7, 11,15. For each beam, the velocity changes with the L-shell angle. If the flows were uniform and L-shell aligned, all points would align with one line corresponding to the average velocity within the KS FoV. For illustration purposes, the line of $-1300 \mathrm{~m} / \mathrm{s}$ is shown.

Fig. 8c). Figure 10 is a series of four KS velocity maps. The first one at 09:30 UT shows more or less smooth variation of the 1-o-s velocity with the azimuth of observations. The only exception is a small "blob" of high velocity in the north-western part of the FoV. The next frame at 09:34 UT is stunningly different as very fast 1-o-s velocities are established in almost half of the field of view. A sharp decrease in the velocity (from red to green color) along the line of central beams 7-8 is easily identifiable. The next frame at 09:38 UT shows a blob of even larger velocity, though limited in space as compared to the previous frame. The velocity receded in other parts of the previously fast flow band. Finally, the velocity map at 09:52 UT shows velocity distri- bution with more smooth variation over the FoV and much smaller overall velocities. Again, a small area of high velocity exists in the north-western part of the FoV. These KS plots seem to indicate the existence of a high-velocity background flow with the occurrence of additional short-lived $(\sim 10 \mathrm{~min})$ blobs/channel of extremely high velocity localized in latitude.

We now illustrate this point differently. In the top panel of Fig. 11 we show a diagram explaining that the measured SuperDARN velocity is the product of the plasma convection velocity $\mathrm{V}_{E}$ and the cosine of the $\mathrm{L}$-shell angle $\Phi$, if the flow is more or less L-shell aligned. It means that if we consider the values of the KS 1-o-s velocity divided by the cosine of 


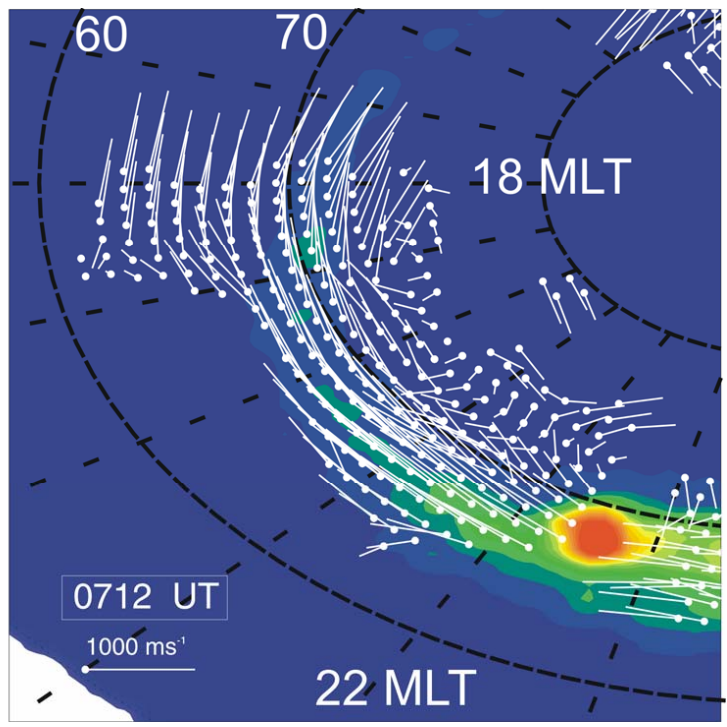

Fig. 12. The same as in Fig. 8 but for observations on 1 December 2001 at 07:12 UT. Fast flow equatorward of the auroral oval is seen in the dusk sector $(\sim 18: 00$ MLT). This flow is comparable in magnitude with the one existing within the auroral oval $\left(\sim 65^{\circ}\right)$ between 18 MLT and 22 MLT.

the L-shell angle at each bin of measurements, we can obtain estimates of the total convection velocity within the FoV. In Fig. 11 we present plots of the obtained velocity versus the L-shell angle for 2-min intervals for the same frames as the ones in Fig. 10. To distinguish data from various beams a special color scheme has been adopted, as follows: black corresponds to data obtained in beams $0,4,8,12$; red corresponds to data in beams $1,5,9,13$; green corresponds to data in beams $2,6,10,14$; and blue corresponds to data in beams $3,7,11,15$.

To understand the meaning and implications of these plots, consider the frame for 09:30-09:32 UT, Fig. 11a. The blue colored dots at L-shell angles of $\sim 75^{\circ}$ (nearly vertical "stripe") are the 1-o-s velocities divided by the cosine of the L-shell angle at each radar bin, as measured in beam 15 over two scans. The velocity spans quite a range of values, and the majority of points are spread between 0 and $-400 \mathrm{~m} / \mathrm{s}$. The next near vertical "stripe" of green dots at L-shell angles of $\sim 70^{\circ}$ corresponds to observations in beam 14 . The data for smaller L-shell angles reflect measurements in smaller number beams, so eventually the black colored dot at the L-shell angle of $\sim 10^{\circ}$ corresponds to observations in beam 0 .

The presented scatter plot (and all others) is complicated, but it will be used only to illustrate some gross features, such as the development of relatively short-lived, high-velocity westward streams on top of fairly fast background flow. Our argumentation is as follows: if the flow were uniform and directed along the L-shells, all points in Fig. 11a would have to cluster around the same value (at various L-shell angles). In Fig. 11a, there are two clusters of points, one around
$-200 \mathrm{~m} / \mathrm{s}$ and another one at $-1300 \mathrm{~m} / \mathrm{s}$ (horizontal line). The first cluster of points reflects the flow at high latitudes, well above the area of interest, and these points will not be discussed below. The other cluster of points reflects the background flow of $\sim-1300 \mathrm{~m} / \mathrm{s}$.

Figure $11 \mathrm{~b}$ shows that the cluster of points around $-1300 \mathrm{~m} / \mathrm{s}$ is split onto two groups; one group of velocities is centered around $-1000 \mathrm{~m} / \mathrm{s}$ while the other group is centered around $-1600 \mathrm{~m} / \mathrm{s}$. We believe that the second group reflects the development of SAPS-like flow within the KS FoV. Figure 11c shows that this second group of velocities is distinctly different from the rest of the points. Some points have velocity of $-2.5 \mathrm{~km} / \mathrm{s}$. We showed in Fig. 8c that the largest velocities were observed at the most equatorward edge of the luminosity band. Finally, Fig. 11d shows only one cluster of points around $-1300 \mathrm{~m} / \mathrm{s}$, indicating the disappearance of the SAPS-like flow enhancement. Thus, the data presented in Figs. 10 and 11 indicate that 1) the plasma flow can be very fast and cover a significant portion of the ionosphere near the equatorward edge of the auroral oval and 2) episodically, very high-velocity, azimuthally-oriented streams can be excited for short periods of 5-10 $\mathrm{min}$.

\section{Comments on other high-velocity King Salmon events}

We studied in detail several other events for which very highvelocity echoes were observed by the KS radar in the afternoon/evening MLT sector and information on luminosity from IMAGE was available. A common feature of these events is that as the oval luminosity decays at the recovery phase of the substorm, a strong westward flow can be established at the equatorward edge of the auroral oval, in the area of weak precipitation (luminosity) and low ionospheric conductance, just as it was demonstrated for the 5 December 2001 event. The time delays between the substorm onset and the establishment of the strong westward flow varied between 10 and $50 \mathrm{~min}$. It is important to note that the flows were not always channels extended over many hours of MLT; instead, often they looked confined to a certain MLT sector in which the luminosity was decreased.

On several occasions, the SuperDARN convection patterns were extended well equatorward of the auroral oval and we were able to observe the flow intensity in these areas. One such example is given in Fig. 12 for the 1 December 2001 event, 07:12 UT. Here fast flow is seen in the dusk sector ( 18:00 MLT), and it extends well equatorward of the oval. The magnitude of the flow outside of the oval is comparable in magnitude to the one existing inside the oval $\left(\sim 65^{\circ}\right.$, 18:00-22:00 MLT). Unfortunately, vectors are not available for even lower latitudes, and it is impossible to investigate how the convection decreases at lower latitudes. This specific case shows that fast flow equatorward of the oval is not always "detached" from the flow within the oval, in contrast with the impression given by $\mathrm{MH}$ observations that strong 


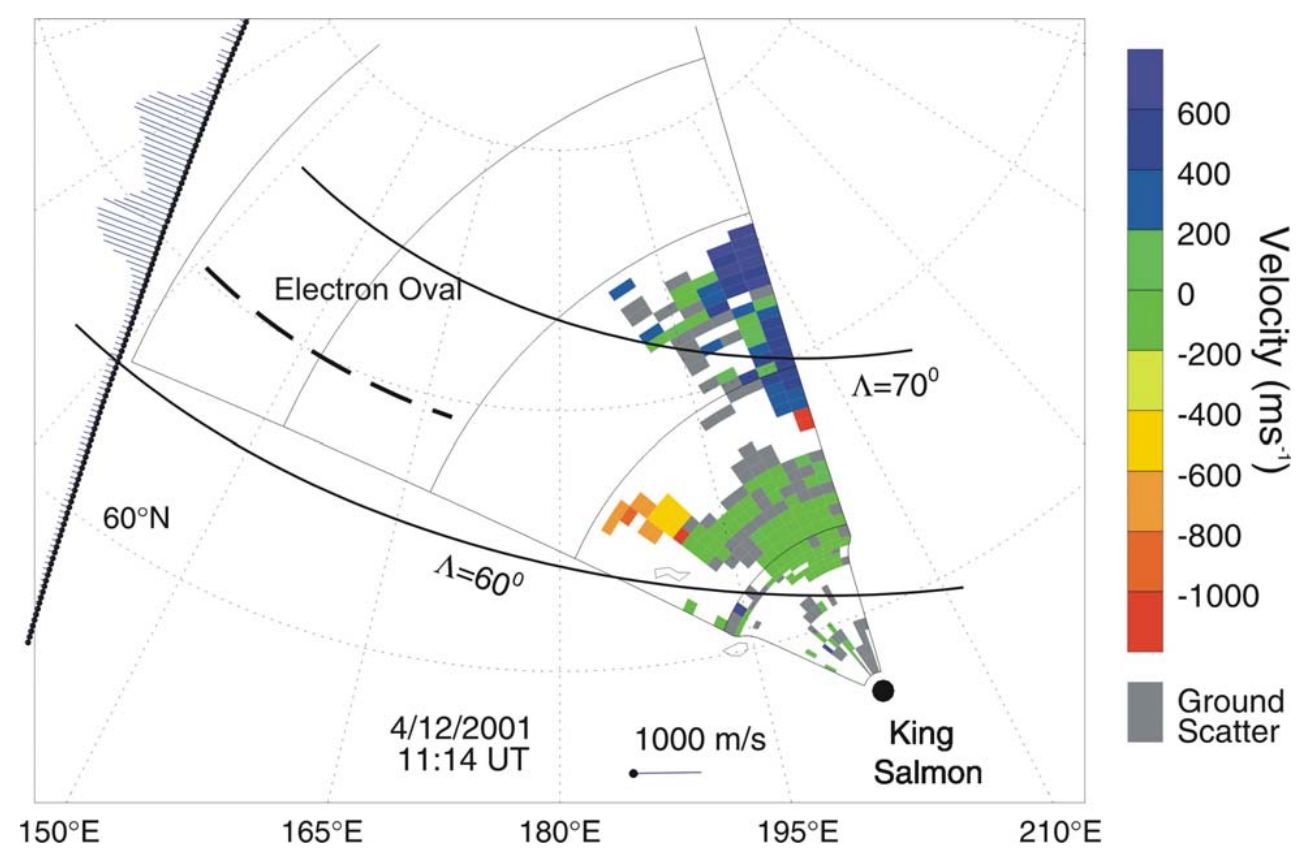

Fig. 13. (a) King Salmon velocity map for 4 December 2001, 11:14 UT with overlaid DMSP cross-track ion drifts for a DMSP F 15 pass near the time of radar measurements. A blob of high velocity KS echoes is clearly identifiable as a red spot. The ion velocity shows two peaks, with one being well outside the auroral oval. The KS high-velocity blob is located at similar latitudes as the equatorward peak seen by DMSP.

flow channels at mid-latitudes are distinctly separated from flows within the oval. In this respect, it has been an important task to identify the KS events with SAPS-like flows that are "detached" from the auroral zone flow. Such attempts were unfortunately unsuccessful. For many high-velocity events, echoes existed only in the low number beams (azimuthally oriented), perhaps because of strong HF radio wave absorption in other beams. For such events, it was impossible to identify the latitudinal trends in the velocity. For some events, it was more or less clear that the monitored fast flow was well equatorward of the auroral oval. Figure 13 gives an example of such observations. Here the DMSP measurements are compared with velocities observed by the KS radar. The DMSP clearly indicate two peaks in the flow, one within the oval and another one well outside it. The KS radar sees regular background flow in most of the FoV. The exception is the blob of high-velocity echoes in beams 3-6. These echoes are located at the same magnetic latitudes as the enhanced flow channel observed by DMSP F15 equatorward of the oval. This example illustrates the difficulties of the KS radar to monitor fast flows; the echoes are limited to a portion of the area where the fast SAPS-like flow is expected.

\section{Discussion}

The data presented in this study show that the King Salmon radar regularly observes high-velocity echoes (1-o-s velocity
$>450 \mathrm{~m} / \mathrm{s}$ ) in the low number beams which are oriented close to the magnetic $\mathrm{L}$ shell directions (the L-shell angles are $\sim 10^{\circ}-20^{\circ}$ ). Such echoes occur mostly in the dusk sector between 16:00 MLT and 23:00 MLT with the largest 1-o-s velocities at about 21:00 MLT (winter) and at a magnetic latitude of $65^{\circ}$. The maximum observed l-o-s velocities are of the order of $2 \mathrm{~km} / \mathrm{s}$; if an assumption on the L-shell alignment of the flow is made, this would correspond to a total velocity of $\sim 2.5 \mathrm{~km} / \mathrm{s}$. Statistically, the largest average 1-o$\mathrm{s}$ velocities are achieved during the winter and the smallest during the summer. For individual scans, the high-velocity echoes cover a range of magnetic latitudes of at least several degrees. In this study, due to the lack of data in some beams in many events, no attempt has been made to determine the exact latitudinal width of the enhanced flow channels. Moreover, for some events, echoes were not detected at all in the high number, nearly poleward-oriented beams, leaving no chance for a channel width determination. The high-velocity echoes are generally observed at relatively low magnetic activity, as characterized by the Kp magnetic index $(\mathrm{Kp}<3-4)$. For stronger magnetic activity, some low range echoes (at magnetic latitudes of $58^{\circ}-60^{\circ}$ ) are still detectable but these are coming from the E-region and their velocity can be well below the $\boldsymbol{E} \times \boldsymbol{B}$ drift so that identification of the SAPS flow is problematic.

The KS observations are reminiscent of the statistical morphology of SAPS flows determined from the Millstone Hill incoherent radar observations at low magnetic activity 
(Foster and Vo, 2002). The similarities between the two statistics are that generally high velocities are seen by both systems and that the velocity maximum is observed in the dusk sector. There are, however, significant differences. An obvious one is that the MH radar sees SAPS more than 4050\% of the time between 20:00-22:00 MLT and at Kp=34 (see Fig. 8 in Foster and Vo, 2002), while detection of the high-velocity echoes by the KS radar is very infrequent. We showed in Fig. 2a that the echo occurrence rate at KS is less than $15 \%$ at the expected latitudes and magnetic local times (15:00-24:00 MLT) of SAPS for December 2001. This makes SAPS detection more difficult, especially in view that for the subsequent years (2002-2004), the echo occurrence at KS went down, in accord with a general decay of the solar activity. We counted the number of high-velocity events (beam 1) for the three months considered in Fig. 2, as well as for June 2002, due to reduced events in the summer, for those days for which the echoes were detected at the expected latitudes and in the magnetic local time sector of SAPS. The threshold velocity was set to $450 \mathrm{~m} / \mathrm{s}$ (similar to Parkinson et al., 2005b). We found that significant high-velocity events were observed in $46 \%$ (winter), 28\% (equinox) and 26\% (summer) of the days with reliable echo detection.

We should mention that Parkinson et al. (2005b) reported larger high-velocity echo occurrence rates for the BI SuperDARN radar in Australia, of the order of $40-50 \%$ (at least "every third night of observations"). These authors considered data in beam \#15 which has the flow angle with L shells of $\sim 50^{\circ}$ (versus $10^{\circ}$ for the low-latitude KS beams). The flow was classified as fast if the 1-o-s velocity was $>450 \mathrm{~m} / \mathrm{s}$. The BI occurrence rates are in agreement with the KS statistics for winter. For equinox and summer, the KS radar detects fewer high-velocity events than the BI radar.

The lower rate of fast flow detection at KS as compared to the $\mathrm{MH}$ observations originates, very likely, from the difference in the radars' capabilities. While the MH radar provides velocity measurements most of the time wherever its beam is pointed, the KS HF radar is very sensitive to the horizontal and vertical electron density distribution in the ionosphere, so that the echo cannot be detected even if irregularities, and fast flows, do exist in the ionosphere. In addition, the KS radar can only reliably observe azimuthal flows at latitudes $62^{\circ}-65^{\circ}$ in a very narrow range of MLT sectors, for individual scans. The echo band for the KS observations is not usually large; more equatorward parts of the ionosphere are not often accessible for echo detection because of low electron density, leading to insufficient radio wave refraction, and for observations deeper into the oval, radio wave absorption is too strong for the echoes to be detected or strong E-region density can over-refract radio waves and blanket echoes from the $\mathrm{F}$ region. This means that a significant portion of the high-latitude ionosphere is simply not accessible for HF measurements. The differences between the KS and BI observations can be explained by the lower sensitivity of the KS radar itself, primarily because of the types of antennas used.
Another important difference in the KS and MH observations is in the average velocity. At 21:00 MLT the KS radar measures speeds up to $750 \mathrm{~m} / \mathrm{s}$ (Fig. 2a) at $\Lambda=65^{\circ}$. Foster and Vo (2002) reported $250 \mathrm{~m} / \mathrm{s}$ (see their Fig. 6) at this latitude, though for stronger magnetic activity of $\mathrm{Kp}=6$.

For these conditions, velocities of $750 \mathrm{~m} / \mathrm{s}$ were observed at the latitude of $\sim 57^{\circ}$, and one might think that the velocity peak was shifted to lower latitudes at larger Kp. Indeed, according to Fig. 3 in Foster and Vo (2002), the SAPS peak should be located at the latitude of $\sim 62^{\circ}$ for $\mathrm{Kp}=4$ and perhaps even at higher latitudes for smaller $\mathrm{Kp}$, meaning that the latitudinal locations of the SAPS peak for the MH and high-velocity KS observations are very comparable. However, with the $\mathrm{Kp}$ decrease the magnitude of the flows also decreases; according to Fig. 5 of Foster and Vo (2002), it changes from $\sim 1000 \mathrm{~m} / \mathrm{s}$ at $\mathrm{Kp}=6$ to $\sim 500 \mathrm{~m} / \mathrm{s}$ at $\mathrm{Kp}=3$. This implies that, generally, the KS velocities are larger than the ones reported by Foster and Vo (2002).

The MH and KS velocities are also different with respect to the time at which their maximum is observed. For $\mathrm{Kp}=4$, the MH maximum (peak) velocities occur at $\sim 16: 00$ 18:00 MLT (see Fig. 3 of Foster and Vo, 2002) while at KS it is at $\sim 21: 00$ MLT for winter and equinox, and there is an additional peak between 16:00-17:00 MLT during summer. The reason for this difference is not clear. We should remind the reader that in this study we considered the l-o-s velocities roughly along the $\mathrm{L}$ shells. The velocities reported by Foster and Vo (2002) included observations at all MH radar azimuths, along and perpendicular to the flow, and the assumption that the flow is L-shell aligned has been made by these authors. It is not clear to what extent such an assumption is applicable to observations at large flow angles. For example, because the large-scale convection cells' foci are often observed at $\sim 18: 00$ MLT, the flow is often round in shape and one hardly can expect it to be L-shell aligned in this time sector. In this respect, it is interesting to note that Ridley and Liemohn (2002) predicted significant departure of SAPS flows from the L-shell directions at $~ 18: 00$ MLT (see their Fig. 6).

Our detailed analysis of the fast flows location with respect to the auroral oval (as seen by IMAGE and DMSP) showed that enhanced flows often occur at the equatorward edge of the auroral oval in the area where the ionospheric conductance is low because of the lack of precipitating particles. Such situations are not rare at the recovery phase of a substorm. Thus, some fast flows seen by the KS radar occur under conditions similar to the ones observed during SAPS events. In this view, such flows can be called SAPS and related to the same production mechanisms as SAPS.

Our attempts to locate events with very narrow jets, as well as strong jets clearly detached from the main flow in the auroral oval, were unsuccessful. This is consistent with the observations by Foster and Vo (2002), who reported the detached channel formation only for magnetically active periods with $\mathrm{Kp}>5$. As we mentioned, for such conditions, 
the expected location of the SAPS flow is equatorward of the KS FoV. Clearly, the recently built low-latitude SuperDARN radars will be in a better position to address this issue.

For the events considered in this study (and several others that we studied), the strong westward flows had a similar pattern of development during the course of a substorm. At the substorm onset time, a clockwise vortex of dusk plasma flow was usually seen. This is consistent with the model of the substorm-related convection pattern development proposed by Grocott et al. (2002). The focus of the vortex was located in the late dusk sector and at latitudes $\sim 70^{\circ}$, slightly poleward and westward of the developing bulge of the auroral luminosity. The vortices often exhibited strong equatorward (meridional) flows at their eastern edge. Some of the near onset-time vortices showed an enhanced westward (azimuthal) flow at their equatorward portion, as well. Similar enhancements of the azimuthal flows have been reported by Provan et al. (2004), in their Fig. 7. These flows well might be the ones noticed by Parkinson et al. (2003a, 2005a, b) at lower magnetic latitudes. Thus, our data support the finding of Parkinson et al. (2003a, 2005a, b) that the westward flow can be enhanced at the substorm onset time in the area equatorward of the expanding auroral bulge. The difference from previous reports is in the timing for the onset of strongest westward flows; we noticed them to occur with a delay of 10-50 min after the substorm onset.

Later on, during the substorm expansion phase, the convection vortices evolve into more large-scale, cell-like flows and eventually disappear. After some time from the onset ( $\sim 40 \mathrm{~min}$ for 5 December 2001) strong and more stable westward/azimuthal flows appear at the equatorward edge of the dusk convection cell in the dusk sector of $\sim 19: 00$ 23:00 MLT. These flows exist for at least 10-20 min, and they overlap with the equatorward edge of the auroral oval. The delay in strong flow development is consistent with satellite detection of strong SAIDs about $30 \mathrm{~min}$ after the substorm onset (Anderson et al., 1993). In this respect, an interesting result was published by Provan et al. (2004). These authors statistically studied the convection pattern evolution in the course of a substorm. By searching through their Fig. 8, we discovered that those data also show an additional enhancement of the azimuthal flow $\sim 14$ min after the substorm onset. Upon our request, the first author of this study (G. Provan) performed a more detailed estimate of the time when the velocity was at maximum (in their paper, only convection patterns at 4, 14, 20 and $28 \mathrm{~min}$ after the onset were presented). It was found that maximum velocities are achieved $\sim 16 \mathrm{~min}$ after the substorm onset. Thus, the delays of 10-50 min that we reported are consistent with the completely different data set (no KS observations) of Provan et al. (2004).

We should note that for the event of 5 December 2001 the fastest flows were observed at the time when the IMF $B_{z}$ had changed polarity to negative values. It might be that such a change activated merging processes in the tail and thus provided stronger convection at the edge of the auroral oval through either energetic particle delivery or a magnetospheric electric field increase and stronger field penetration outside the oval. However, we note that the velocity in beam 1, Figs. 4 and 5a, was persistently increasing in magnitude well before the $B_{z}$ changes occurred. It even seems that the $B_{z}$ transition did not change the temporal trend of the azimuthal velocity.

The data presented in this study show that the fast flows vary in velocity magnitude quite quickly. We showed in Fig. 11 the development of short-lived, very fast streams (up to $2.5 \mathrm{~km} / \mathrm{s}$ ) over the period of several minutes. These existed on top of the background azimuthal flow of $\sim 1.3 \mathrm{~km} / \mathrm{s}$, which, by itself, is unusually fast. Our data are similar to observations by Erickson et al. (2002) and Foster et al. (2005), who reported the high variability of the flow within the SAPS channel by looking at the E-region power data underneath the SAPS flow in the F region.

In terms of the mechanism of high-velocity flow formation, our data support a notion that strong westward flow channels near the equatorward edge of the auroral oval develop when the Region 2 FAC flows into the ionosphere with low conductance, due to the lack of precipitation there (e.g. Foster and Burke, 2002). Such conditions frequently occur at the recovery phase of the substorm in the equatorial part of the auroral oval where precipitation suddenly ceases to exist. A decrease in the precipitation intensity happens with various delays after the substorm onset, and it is not surprising that high-velocity flows detected by the KS radar have different delays from the onset time, ranging from about $10 \mathrm{~min}$ to $50 \mathrm{~min}$. It might well be that strong plasma flows near the equatorward edge of the auroral oval are enhanced by energetic protons penetrating into the inner magnetosphere, as suggested by Galperin (2002). The data available do not allow us to evaluate the relative importance of each of these factors.

\section{Conclusions}

In this study we showed that:

1. The King Salmon SuperDARN radar regularly detects enhanced flows (velocity $>450 \mathrm{~m} / \mathrm{s}$ ) roughly along the magnetic L-shell directions at magnetic latitudes of $60^{\circ}-65^{\circ}$ and in the local time sector of 16:0023:00 MLT. The flows are seen either as a broad band of echoes covering several degrees of magnetic latitude or echoes in several L-shell aligned beams corresponding to $\sim 1^{\circ}-2^{\circ}$ of magnetic latitude. The enhanced flows are seen $\sim 30 \%$ of the echo detection time and at relatively low magnetic activity of $\mathrm{Kp}<3-4$. In individual events, the line-of-sight velocity can be as high as $2 \mathrm{~km} / \mathrm{s}$, which is in the range of maximum velocities seen with any $\mathrm{Su}-$ perDARN radar. There are seasonal trends in the average velocity of the flow; velocities are largest (smallest) 
for the winter (summer) conditions. The maximum velocities are observed around 21:00 MLT during winter and equinox, and there is an additional peak at 16:0017:00 MLT during summer.

2. For a number of identified fast flow events, the velocity maximum coincides with the equatorward edge of the auroral oval/precipitation, as inferred from the DMSP and IMAGE particle data. Exceptionally high velocities were observed in the areas of low local precipitation.

3. The band of high-velocity echoes can consist of shortlived (5-10 min) streams with exceptionally high velocity which occurs in the areas of low precipitation.

4. For a number of analyzed events, the fast flows occur at the recovery phase of a substorm developing in the area of observations. The velocity of the westward flow starts to increase near the substorm onset time, in agreement with previous coherent radar studies, but the maximum velocities are achieved later, 10-50 min after the onset. The enhanced flows constitute the equatorward part of the large-scale dusk convection cell in the 19:0023:00 MLT sector. The westward velocity enhancement occurs after the substorm-related large-scale convection vortex, centered typically near the westward edge of the auroral luminosity bulge, evolves into larger-scale celllike flow. Also, the fastest flows are seen with some delay from the arrival of energetic protons to the geostationary satellite located in the duskside magnetosphere.

5. The observations indicate that at least some of the observed high-velocity westward flows seen by the KS radar can have the same origin as subauroral polarization streams, and thus this radar is a useful instrument for studying these streams at low magnetic activity.

Acknowledgements. We acknowledge the support of all national funding agencies that made possible the continuous operation of the SuperDARN radars considered in this study. The DMSP ion drift data were taken from the University of Texas at Dallas website (M. Hairston). The DMSP particle data were taken from the APL website (P. Newell). We thank F. Rich (Air Force Research Laboratory) for providing access to the DMSP data on the AFRL website; this enormously helped us in events search. LANL particle data were taken from the CDAWeb site (E. Dors). We thank G. D. Reeves for comments on LANL satellite particle data. Helpful discussions on the topic of the research with E. V. Mishin, G. Khazanov, and S. Sazykin are appreciated. We thank G. Provan for estimates of the maximum convection velocities during substorm. This work has been supported by NSERC (Canada) grant to AVK.

Topical Editor M. Pinnock thanks H. Vo and another referee for their help in evaluating this paper.

\section{References}

Anderson, P. C., Heelis, R. A., and Hanson, W. B.: The ionospheric signatures of rapid subauroral ion drifts, J. Geophys. Res., 96, 5785-5792, 1991.

Anderson, P. C., Hanson, W. B., Heelis, R. A., Craven, J. D., Baker, B. N., and Frank, L. A.: A proposed production model of rapid subauroral ion drifts and their relationship to substorm evolution, J. Geophys. Res., 98, 6069-6078, 1993.

Anderson, P. C., Carpenter, D. L., Tsuruda, K., Mukai, T., and Rich, F. J.: Multisatellite observations of rapid subauroral ion drifts (SAID), J. Geophys. Res., 106, 29 585-29 599, 2001.

Bristow, W. A., Otto, A., and Lummerzheim, D.: Substorm convection patterns observed by the Super Dual Auroral Radar Network, J. Geophys. Res., 106, 24 593-24 609, 2001.

Bristow, W. A., Sofko, G. J., Stenbaek-Nielsen, H. C., Wei, S., Lummerzheim, D., and Otto, A.: Detailed analysis of substorm observations using SuperDARN, UVI, ground-based magnetometers, and all-sky imagers, J. Geophys. Res., 108, doi:10.1029/2002JA009242, 2003.

Carpenter, D. L. and Lemaire, J.: The plasmasphere boundary layer, Ann. Geophys., 22, 4291-4298, 2004.

Erickson, P. J., Foster, J. C., and Holt, J. M.: Inferred electric field variability in the polarization jet from Millstone Hill E region coherent scatter observations, Radio Sci., 37, 1027, doi:10.1029/2000RS002531, 2002.

Foster, J. C. and Burke, W. J.: SAPS: A new characterization for sub-auroral electric fields, EOS Trans. AGU, 83, 393-395, 2002.

Foster, J. C. and Vo, H. B.: Average characteristics and activity dependence of the subauroral polarization stream, J. Geophys. Res., 107, doi:10.1029/2002JA009409, 2002.

Foster, J. C., Erickson, P. J., Lind, F. D., and Rideout, W.: Millstone Hill coherent-scatter radar observations of electric field variability in the sub-auroral polarization stream, Geophys. Res. Lett., 31, L21803, doi:10.1029/2004GL021271, 2005.

Galperin, Y. I., Ponomarov, Y. N., and Zosimova, A. G.: Direct measurements of ion drift velocity in the upper ionosphere during a magnetic storm, Cosmicheskie Issled., 11, 273-283, 1973.

Galperin, Y. I.: Polarization jet: Characteristics and a model, Ann. Geophys., 20, 391-404, 2002.

Greenwald, R. A., Baker, K. B., Dudeney, J. R., Pinnock, M., Jones, T. B., Thomas, E. C., Villain, J.-P., Cerisier, J.-C., Senior, C., Hanuise, C., Hunsuker, R. D., Sofko, G., Koehler, J., Nielsen, E., Pellinen, R., Walker, A. D. M., Sato, N., and Yamagishi, H.: DARN/SuperDARN: A global view of the dynamics of highlatitude convection, Space Sci. Rev., 71, 763-796, 1995.

Grocott, A., Cowley, S. W. H., Sigwarth, J. B., Watermann, J. F. and Yeoman, T. K.: Excitation of twin-vortex flow in the nightside high-latitude ionosphere during an isolated substorm, Ann. Geophys., 20, 1577-1601, 2002.

Hamza, A. M., Huber, M., Lyatsky, W., Kustov, A. V., Andre, D. and Sofko, G.: Eastward convection jet at the poleward boundary of the nightside auroral oval, Geophys. Res. Lett., 17, 28092812, 2000.

Karlsson, T., Marklund, G. T., Blomberg, L. G., and Malkki, A.: Subauroral electric fields observed by the Freja satellite: A statistical study, J. Geophys. Res., 103, 4327-4341, 1998.

Koustov, A. V., Sofko, G. J., Andre, D., Danskin, D. W., and Benkevitch, L.V.: Seasonal variation of HF radar F region echo occurrence in the midnight sector, J. Geophys. Res., 109, A06305, 
doi:10.1029/2003JA010337, 2004.

Koustov, A. V., Danskin, D. W., Makarevitch, R. A., and Gorin, J. D.: On the relationship between the velocity of E-region HF echoes and ExB plasma drift, Ann. Geophys., 23, 371-378, 2005.

Lacroix, P. J. and Moorcroft, D. R.: Ion-acoustic HF radar echoes at high latitudes and far ranges, J. Geophys. Res., 106, $29091-$ 29 103, 2001.

Parkinson, M. L., Devlin, J. C., Ye, H., Waters, C. J., Dyson, P. L., Breed, A. M., and Morris, R. J.: On the occurrence and motion of decameter-scale irregularities in the sub-auroral, auroral, and polar cap ionosphere, Ann. Geophys., 21, 1847-1868, 2003a.

Parkinson, M. L., Pinnock, M., Ye, H., Hairston, M. R., Devlin, J. C., Dyson, P. L., Morris, R. J., and Ponomarenko, P.: On the lifetime and extent of an auroral westward flow channel (AWFC) observed during a magnetospheric substorm, Ann. Geophys., 21, 893-913, 2003b.

Parkinson, M. L., Dyson, P. L., and Pinnock, M.: The importance of auroral westward flow channels in substorm evolution, Advances in Space Research, doi:10.1016/j.asr.2005.08.028, in press, 2005a.
Parkinson, M. L., Pinnock, M., Wild, J., Lester, M., Yeoman, T. K., Milan, S. E., Ye, H., Devlin, J. C., Frey, H. U., and Kikuchi, T.: Interhemispheric asymmetries in the occurrence of magnetically conjugate sub-auroral polarization streams, Ann. Geophys., 23, 1371-1390, 2005b.

Provan, G., Lester, M., Mende, S. B., and Milan, S. E.: Statistical study of high-latitude plasma flow during magnetospheric substorms, Ann. Geophys., 22, 3607-3624, 2004.

Ridley, A. J. and Liemohn, M. W.: A model-derived storm time asymmetric ring current driven electric field description, J. Geophys. Res., 107, 1151, doi:10.1029/2001JA000051, 2002.

Ruohoniemi, J. M. and Greenwald, R. A.: Rates of scattering occurrence in routine HF radar observations during solar cycle maximum, Radio Sci., 32, 1051-1070, 1997.

Ruohoniemi, J. M. and Baker, K. B.: Large-scale imaging of highlatitude convection with Super Dual Auroral Radar Network HF radar observations, J. Geophys. Res., 103, 20 797-20 811, 1998.

Spiro, R. W., Heelis, R. A., and Hanson, W. B.: Rapid subauroral drifts observed by Atmospheric Explorer C, Geophys. Res. Lett., 6, 657-660, 1979.

Yeh, H. C., Foster, J. C., Rich, F. J., and Swider, W.: Storm time electric field penetration observed at mid-latitude, J. Geophys. Res., 96, 5707-5721, 1991. 